\title{
AWD, Cholera Outbreak / Children of Somalia
}

\author{
Hamid Yahya Hussain* \\ Department of Community and Family Medicine, UAE
}

*Corresponding author: Hamid Yahya Hussain, Department of Community and Family Medicine, WHO public health Medicine Consultant, P O BOX 23317, Sharjah, UAE

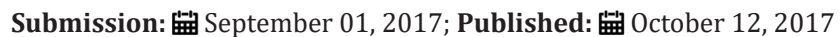

\section{Editorial}

In June 2017, I have Joined World health organization mission to Somalia in responding efforts to AWD/Cholera ongoing outbreak, the long run instability of the country has led to the continuous deterioration of the sanitation and safe-water infrastructure. Only 10 per cent of the rural population and 63 per cent of the urban population has access to improved drinking water it is no strange that acute watery diarrhea (AWD) and cholera are widely disseminated among children population of Somalia which necessitate focusing of international efforts to help those victims. There is no surprise that morbidities and mortalities rates are upraising significantly.

The World Health Organization (WHO) has attempted to reduce the impact of AWD through adopting defend intervention strategies, National health care system capacity building (training of health care professionals on standards cases management), (strengthening patient assessment, categorization and triage system, developing patient developing adequate and effective patient transfer system, and patient referral system for AWD/ non cholera cases . A major gap has been identified at Infection prevention and control measures. Community social mobilization efforts operating powerfully, Environmental sanitation, adequate safe water supply, hygiene practice at personal, household and environmental level were addressed.

The high investing in hand hygiene practice among general population were stressed on, utilization of latrines and many other gaps were accordingly followed. Both at health care facilities and at community based level by developing local capacity, and this course which seems to be working. The WHO is focusing their efforts on strengthening coordination between local health actors, early disease detection and training health care workers. This is allowing for a timely response to outbreaks. Nonetheless, the lack of sanitation and safe-water infrastructure will continue to compromise the health of Somalis and promote conditions where cholera and AWD outbreaks are possible, even likely. Security alone will not address the health needs of Somalia; a secure environment is a necessary condition for building a health system that can begin to address the many needs of the population. The WHO has recorded a reduced incidence of AWD and are assisting in the establishment of a well-planned, if basic, local health system 